\title{
Contexto y procesos cognitivos. La interacción niño-adulto
}

\author{
PILAR LACASA \\ PILAR HERRANZ \\ Universidad Nacional de Educación a Distancia

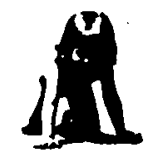 \\ Resumen
}

En este trabajo se revisa la incidencia del contexto social en el desarrollo cognitivo del niño. En él se analiza este problema desde diferentes perspectivas teóricas inspiradas en la biologia y la etología, y se alude al planteamiento ecológico de Bronfenbrenner. Se presenta especial atención a los trabajos empiricos que, partiendo de la obra de Vygotsky, analizan la relación del niño con el adulto insistiendo en cómo ésta desempeña un importante papel en el paso de la regulación de la propia conducta a partir de los otros a la autorregulación y en cómo facilita al niño, en situaciones de aprendizaje o de solución de problemas, formas adecuadas de codificación o de estructuración de las tareas.

Palabras clave: Contexto, Desarrollo Cognitivo, Aprendizaje, Habla privada, Regulación, Planificación.

\section{Context and cognitive processes. Adult-child interaction}

\section{Abstract}

The approach proposed in this paper aims for an integration of context and cognitive development. Children adapt to the constrains and adopt the techniques provided by society through practice and instrucion with other people guiding their activities. Several theoretical frameworks are presented, taken from Biology, Ethology and Ecology. Our reflection about the problem is close to the theoretical perspective that emphasizes in the sociocultural dimension of the context. Several authors have attempted to show that adult-child interaction is a necessary precursor to strategic skill in young children. The researcb we have reviewed allow us to conclude that the adult does not only improve children's learning in specific tasks, but facilitates more complex processes in which the child learns to learn.

Keywords: Context, Cognitive Development, Leaming, Private Speech, Regulation, Planning Skills.

Agradecimientos: Agradecemos a Amelia Alvarez y Pablo del Río sus comentarios y sugerencias para la preparación de este trabajo.

Dirección del autor: UNED, Facultad de Psicología, Departamento de Psicología Evolutiva, Ciudad Universitaria, s/n, 28040 Madrid.

Original recibido: Septiembre 1988. Revisión recibida: Diciembre 1988. Aceptado: Diciembre 1988. 
Tradicionalmente la piscología ha estado interesada en examinar la conducta y la vida mental del individuo pero, aun reconociendo que éstas son inseparables del contexto en el que aparecen, no siempre se ha analizado en profundidad el papel de éste en la génesis y desarrollo del comportamiento. Estudios recientes han mostrado, sin embargo, que las actividades cognitivas fluctuan en función de la situación. Se señala, por ejemplo, que el rendimiento del niño en problemas que parecen exigir un mismo tipo de procesos de conocimiento varía en relación con aspectos concretos de la situación en la que se realiza una tarea, por ejemplo, la familiaridad con el problema (Gelman, 1978; DeLoache, 1980), el tipo de materiales que se utilizan (Kingma, 1984; Lacasa y otros, 1985), o el hecho de interactuar con unas personas u otras (Rogoff, 1982; Ellis y Rogoff, 1986). El objetivo de esta exposición es analizar el papel del contexto social, concretamente de la interacción del niño con el adulto, en el desarrollo cognitivo. Revisaremos el tema desde diversas perspectivas teóricas en las que están implicadas diferentes nociones de contexto que, por otra parte, no siempre coinciden en el peso que se le asigna como un factor del desarrollo.

\section{DIFERENTES APROXIMACIONES TEORICAS AL ESTUDIO DEL. CONTEXTO}

Sería muy difícil proponer una definición de contexto que aglutinara sus múltiples aspectos y en la que, además, estuvieran de acuerdo los investigadores. Esto se debe, sobre todo, a dos razones. Por una parte, conviene señalar que cuando se adopta una determinada idea acerca de lo que es el contexto ésta suele estar en estrecha relación con los presupuestos teóricos asumidos. Por otra parte, aquellas teorías en las que el contexto juega un importante papel en la explicación de la conducta, suelen situarse en una perspectiva interdisciplinar y los psicólogos buscan modelos inspirados, por ejemplo, en la biología (Piaget, 1967; Samerof, 1982), la etología. (Bowlby, 1969; Hinde, 1983) la ecología (Bronfenbrenner, 1979) o la antropología (Cole, 1975; Cole y Griffin, 1980). Aludiremos ahora a las diferentes nociones del contexto que están implícitas en estos modelos para abordar, desde ellas, el tema de la interación niño-adulto. Aceptar un modelo u otro condiciona tanto el tipo de cuestiones que se plantean como los métodos propuestos para abordarlos. Dos advertencias previas son necesarias. La primera, que los modelos teóricos que presentamos son, únicamente, un ejemplo de los posibles modos de entender las relaciones entre el contexto y los procesos cognitivos, pero en ningún caso agotan los planteamientos que se han ocupado en la actualidad de este tema en psicología del desarrollo. La segunda, que nos detendremos especialmente en aquellos trabajos que ponen el acento en las dimensiones socioculturales del contexto y se refieren al funcionamiento cognitivo del niño en la etapa preoperatoria, por ser este momento del desarrollo el que ha recibido, quizás, una mayor atención por parte de los investigadores.

\section{La.influencia de la biología en el estudio de los procesos cognitivos}

A los psicólogos que se han inspirado en la biología lo que les interesa resaltar, sobre todo, es que el contexto presenta a los individuos determi- 
nadas dificultades que será necesario superar para poder progresar y lograr formas de conocimiento cada vez más complejas. El progreso sólo es posible a través del conflicto, vienen a decirnos. (Sameroff, 1983) es, tal vez, uno de los autores que plantea más claramente el tema del contexto a partir de las aportaciones de la biología considerando, además, la influencia que sobre ésta ha tenido la teoría general de sistemas (Waddington, 1976). En su opinión Piaget es uno de los autores que han adoptado más claramente este modelo, su teoría de la equilibración es una buena muestra de ello (Lacasa, 1984). El contexto se entiende allí como un entorno al que es necesario adaptarse y se presenta como una fuente de dificultades que es preciso superar. Sin embargo, llega un momento en que la evolución prescinde del contexto; no olvidemos que para Piaget la posibilidad de ir más allá de las condiciones espacio-temporales que delimitan las situaciones a las que se enfrenta el sujeto es la característica más propia de la inteligencia.

Desde esta perspectiva cabe resaltar algunas investigaciones que se han ocupado con detalle de la construcción social de la inteligencia, especialmente de la interacción del niño con sus iguales y, en algunos casos, con el adulto. Los trabajos de la escuela de Ginebra (Doise, 1983; 1985; Perret Clermont, 1984) señalan que el progreso en el conocimiento es el resultado de la participación en situaciones de interacción que se apoyan en la existencia de un conflicto sociocognitivo, el papel del adulto está relacionado con la aparición de este conflicto. (Doise y Mugny, 1981) presentan diversas experiencias en relación con este tema. Por ejemplo, en una tarea relacionada con la conservación de longitudes analizan el efecto de diferentes tipos de conflicto sociocognitivo que el adulto puede crear en el niño, utilizando la contra-sugerencia a las respuestas infantiles como técnica de interrogación. Comparan la eficacia de dos situaciones cuando se trata de que los niños progresen en la solución del problema: una de ellas consiste en que el adulto proporciona la solución correcta, que está en contradicción con la respuesta que el niño había dado previamente; en la otra situación, por el contrario, se ofrecen al niño respuestas incorrectas pero que son diferentes de las que el niño había expresado. Los resultados del trabajo mostraron progreso en ambas situaciones. Pensamos, sin embargo, que son necesarios otros estudios que precisen, con mayor exactitud, cual de las dos situaciones resulta más eficaz. En esta línea otros investigadores inspirados en las teorías del aprendizaje social (Bandura, 1977; Zimmerman, 1983) han analizado la incidencia de los modelos sociales en el desarrollo y se critica (Russell, 1981, 1983) el recurso a la presencia de conflictos sociocognitivos como la única causa del cambio. Desde esta perspectiva algunos trabajos (Murray, 1983; Robert, 1983; Robert y Charbonneau, 1977a, 1977b) han señalado la importancia de la interacción con el adulto o con otros niños más competentes en cuanto que proporcionan modelos de conducta que favorecen el desarrollo.

\section{Los planteamientos etológicos}

Estas investigaciones tienen sus raíces en los estudios de Lorenz (1981) y Tinbergen (1972). F. López (1983) ha enumerado con acierto los múltiples temas de estudio que están presentes en ella. Nos interesan ahora las que se relacionan con el desarrollo cognitivo (Charlesworth, 1978, 1983; 
Etienne, 1984). Estos psicólogos consideran también que el conocimiento humano es una forma de adaptación al medio. Aun con el riesgo de simplificar demasiado diremos que sus trabajos se diferencian de los que se realizan desde la perspectiva del modelo anterior, principalmente, por sus aportaciones metodológicas: por una parte, se ocupan de unidades observacionales más amplias que incluyen tanto a la conducta como a las dimensiones del contexto y, por otra, las investigaciones se plantean en situaciones naturales y no de labortorio. Cuando se analizan procesos cognitivos el núcleo conceptual en el que convergen dichas unidades observacionales es la noción de problema. Un problema se define en relación con «un bloque» de la conducta del sujeto que elicita una respuesta observable la cual, a su vez, puede considerarse mediatizada por un proceso cognitivo.

En esta línea de trabajo Charlesworth (1983) cita algunos estudios, que él y su equipo han llevado a cabo, en los que se observan situaciones de interacción niño-adulto. En uno de ellos, por ejemplo, se analiza la relación de un grupo de niños preescolares con el maestro, en conductas de solución de problemas que surgen durante las actividades ordinarias de la escuela. El análisis se centra en el tipo de preguntas y respuestas que emiten los que intervienen en una situación. Los resultados del estudio mostraron que las cuestiones variaban en función del número de sujetos que interactuaban, los niños preguntaban más cuando estaban sólos con el adulto que cuando estaban en grupo. También las respuestas del adulto eran más abundantes en situaciones de interacción individual. Desde nuestro punto de vista, lo que interesa resaltar sobre todo de este estudio es que no se pretende dar una explicación del progreso cognitivo o del modo en que éste se ve facilitado por la interacción del niño con el adulto, tal como ocurría en los trabajos citados anteriormente, sino, más bien, de describir situaciones de la vida diaria en las que se establecen relaciones sociales para resolver determinados problemas.

\section{Entorno social y desarrollo de la conducta: el modelo ecológico}

Otro camino para acercarse a las relaciones que el niño mantiene con el entorno es el que ha seguido el modelo ecológico (Bronfenbrenner, 1979; Bronfenbrenner y Crouter, 1983). Bronfenbrenner, según él mismo dice, pretende abordar determinadas cuestiones que no reciben un tratamiento exhaustivo o que se olvidan en los modelos clásicos de Piaget, Freud o, incluso, Vygotsky; considera, por ejemplo, que no se habrian observado suficientemente las actividades cognitivas de los padres o de otros agentes sociales que se realizan ante el niño ni, tampoco, se habría analizado en profundidad cómo las actividades de socialización, vistas en términos cognitivos, están distribuidas a través de diferentes entornos y relacionadas con la clase social, la etnia o la estructura familiar. Estos problemas son, en su opinión, un tema pendiente de la psicología evolutiva. Para estudiarlos se proponen diferentes niveles de análisis, centrados más en los procesos sociales que en la estructura social. No podemos presentar ahora en una exposición detallada de ellos que, por otra parte, ya existe en castellano (Del Río y Alvarez, 1985) y nos limitaremos a una breve alusión insistiendo en como se plantea, desde esta perspectiva, el tema de la interacción del niño con el adulto. 
El modelo ecológico señala que el análisis del contexto puede ser realizado desde un triple nivel: 1) El microsistema, que alude al entorno más inmediato al niño y a las actividades que tienen lugar dentro de él. Las situaciones a partir de las cuales se define pueden ser consideradas «escenarios» (settings). Incluyen aspectos tan diferentes como, por ejemplo, los objetos físicos y el modo en que el entorno está organizado por los adultos, física y temporalmente $o$, también, las estructuras interpersonales en las que el niño llega a verse comprometido con otras personas. 2) El mesosistema, desde el que se analiza cómo dos o más escenarios pueden afectar a la conducta del niño simultáneamente. 3) El exosistema que se refiere al «contexto externo» del desarrollo humano.

Lo que nos interesa resaltar, especialmente, es que desde cada uno de estos tres niveles de análisis se han realizado investigaciones relacionadas con la interacción niño-adulto. Citaremos algunos ejemplos. Bronfenbrenner (1974), situándose desde la perspectiva del microsistema, estudio la relación del niño con un adulto, por ejemplo la madre, a través del efecto que sobre ella pueda ejercer una tercera persona, por ejemplo el padre. Otro tipo de trabajos son los que comparan las relaciones que mantienen los niños y adultos en varios escenarios: en unos casos se analizan las relaciones que un mismo niño mantiene en dos escenarios distintos, por ejemplo, en casa y en la guardería (Cochran y Robinson, 1982; Gunnarson, 1978); en otros casos los nexos entre escenarios se establecen a través de la figura del adulto (Bronfenbrenner, 1983). Por último, se revisa la influencia que los cambios que se producen en el contexto general de la sociedad ejercen sobre desarrollo del niño. También en este caso hay que tener en cuenta su relación con el adulto. Un ejemplo de estas investigaciones son las que se realizan para analizar las diferencias que producen en los niños el status de trabajo de la madre (Hoffman, 1980; Bronfenbrenner, Alvarez y Enderson, 1982).

Como conclusión de esta breve exposición diremos que las aportaciones de estos trabajos, en nuestra opinión, están más relacionadas con los análisis del entorno social en sí mismo que con observaciones detalladas y minuciosas de los procesos cognitivos del sujeto en los que aquél incide.

\section{El estudio del contexto desde una perspectiva socio-cultural}

Las relaciones entre cultura y conocimiento tienen un carácter peculiar. Es fácil advertir que las manifiestaciones culturales, por ejemplo el arte, los mitos o las creencias, son productos de procesos de conocimiento y que éstos, a su vez, están inmersos en una cultura. Es indudable que no todos los psicólogos son conscientes de ello y que en muchas ocasiones las manifestaciones culturales y el conocimiento individual se han analizado como fenómenos independientes que se estudian con metodología distintas. En el extremo opuesto están los psicólogos que analizan el desarrollo cognitivo en relación con el contexto cultural (Hichmann, 1987; Sinha, 1988; Wertsch, 1981 ; 1985) posiciones que muestran una fuerte influencia de la antropología, ello no resulta extraño ya que esta disciplina se ha visto obligada a dar cuenta de un hecho decisivo: las sociedades proporcionan a sus miembros diferentes tipos de experiencia que condicionan su conocimiento. 


\section{¿Cómo incide el contexto en el conocimiento?}

Para dar razón de la interacción entre los procesos cognitivos y el contexto cultural, en cuanto que éste contribuye a configurar a aquellos, se han dado dos tipos de explicaciones, (Laboratory of Comparative Human Cognition, 1983). La cuestión, ante la que es necesario tomar partido, y a partir de la cual es posible diferenciar esos dos planteamientos, es la de aceptar o no la existencia de "universales cognitivos» a los que el contexto cultural condicionaría y actualizaría. M. Cole y sus colaboradores distinguen claramente esas dos posiciones teóricas y las representan gráficamente. La figura 1 presenta los elementos esenciales incluidos en las explicaciones que aceptan «universales cognitivos», estructuras o incluso procesos a los que podrían tener acceso todos los humanos, mediatizados por el contexto cultural. Desde este punto de vista se asume, por una parte, que las experiencias operan sobre un procesador central que a su vez controla el logro en un determinado conjunto de tareas y, por otra parte, que pueden existir diferencias entre los procesos cognitivos de los individuos que pertenecen a diferentes culturas en función de un determinado grado de evolución de estas últimas. Estos principios serían compatibles, por ejemplo, con la explicación piagetiana de las diferencias encontradas en el rendimiento de lo sujetos a raíz de los estudios transculturales.

FIGURA 1

\begin{tabular}{|c|c|c|}
\hline Suceso & $\begin{array}{c}\text { Sistema } \\
\text { de Procesamiento }\end{array}$ & $\begin{array}{c}\text { Rendimiento en } \\
\text { la tarea }\end{array}$ \\
\hline
\end{tabular}

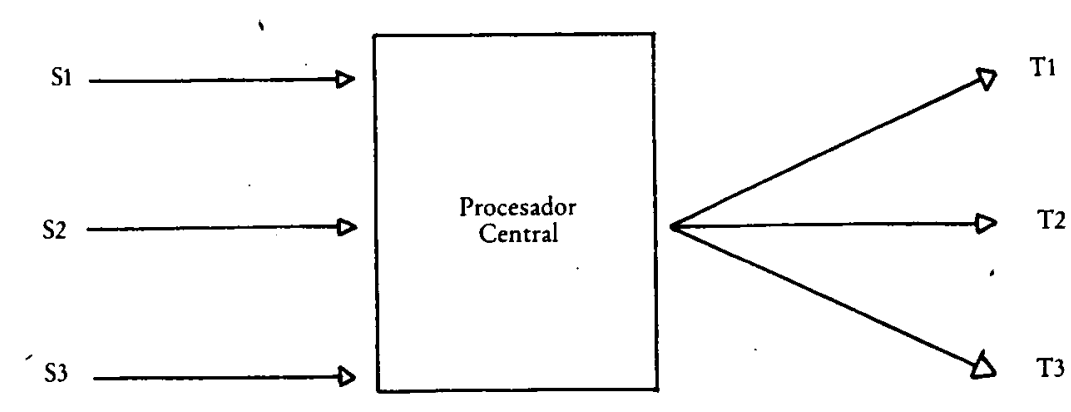

Contexto y procesos cognitivos: universales cognitivos

La figura 2, por el contrario, muestra lo que se considera un procesador distribuido. En este caso se prescinde de un sistema de procesamiento central y el funcionamiento cognitivo se entiende como un "proceso distribuido" a través de los individuos y de las sociedades. Es decir, un mejor rendimiento del sujeto en determinadas tareas, que se produce como consecuencia del desarrollo o del aprendizaje, es támbién función de su experiencia en esa tarea, dicha experiencia está en relación, por lo tanto, con procesos cognitivos específicos que el sujeto actualiza en ciertos contextos. Debemos tener en cuenta, por otra parte, que la cultura en la que el individuo se ve inmerso determina, en gran medida, la posibilidad de que el sujeto 
esté en contacto con un contexto u otro. El principal problema al que se enfrentan este tipo de teorías es explicar la generalización de lo aprendido en nuevos contextos. Muchos de los trabajos que se han inspirado en la psicología de Vygotsky $(1978 ; 1986)$, estableciendo estrechas conexiones entre el contexto socio-cultural y los procesos cognitivos aceptan este planteamiento teórico, revisaremos a continuación cómo el problema de la interacción niño-adulto adquiere en ellos una significación especial.

FIGURA 2

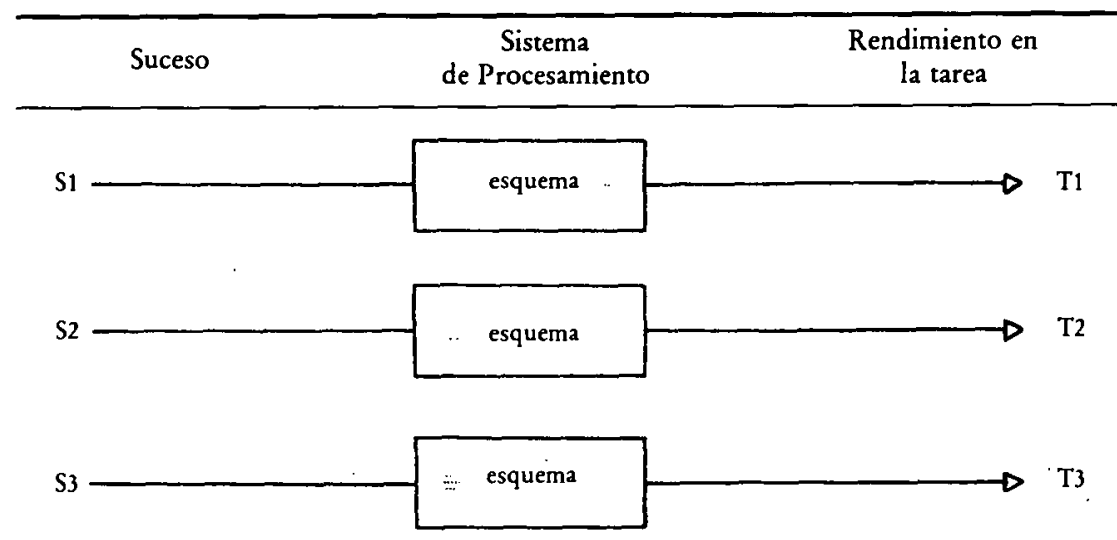

Contexto y procesos cognitivos: procesamiento distribuido

\section{Los fundamentos vygotskianos de la perspectivas socio-cultural}

Quienes se sitúan en esta perspectiva teórica ponen el acento en las dimensiones sociales del contexto y consideran que este transciende el entorno físico y está determinado, sobre todo, por quienes participan en una situación:

«Como McDermott y Roth (1978) han apuntado, el contexto está constituido por lo que las personas están haciéndo tanto como por cuándo y dónde lo hacen. Ello significa que las personas en interacción sirven como entorno a los demás» (Laboratory of Comparative Human Cognition, 1983, p. 333):

El contexto, en este sentido, condiciona profundamente el desarrollo cognitivo. La teoría de Vygotsky $(1978 ; 1986)$ y Leontiev $(1981 ; 1983)$ enfatizan que la actividad cognitiva no puede ser concebida como una característica de la persona independiente del contexto en que esta piensa y actúa, por el contrario, se ve determinada por el entorno sociocultural en dos niveles: por una parte, la interacción social proporciona al niño información y herramientas útiles para desenvolverse en el mundo, esas son transmitidas a los niños a través de los miembros más experimentados de la sociedad; por otra parte, el contexto histórico y socio-cultural controla el proceso a través del cual los miembros de un grupo social acceden a unas herramientas u otras. Aludiremos, brevemente a estos dos niveles.

En primer lugar hay que señalar que, de acuerdo con los planteamientos más clásicos de la psicología soviética (Vygotsky, 1978), las funciones 
cognitivas aparecen primero en el plano social (interpsíquico) y sólo después en el nivel individual (intrapsíquico). El concepto de "zona del desarrollo próximo "ayuda a comprender este proceso. Con él se indica, esencialmente, que los niños pueden hacer con un adulto o con un igual más capaz lo que no son capaces de lograr solos. Por tanto, la zona de desarrollo próximo es la distancia entre la capacidad de resolver un problema independientemente y la capacidad de solucionarlo junto a un adulto u otro miembro más capaz. Así, el desarrollo cognitivo ocurre en situaciones donde la solución del problema del niño es guiada por el adulto, en la zona de desarrollo próximo, regulando su actividad y proporcionándole un modelo de ejecución maduro.

En segundo lugar, la cultura maximiza su impacto sobre el desarrollo proporcionando contextos a los que el niño accede en función de la zona de dearrollo próximo. Desde este punto de vista se reconoce que existen varios caminos a través de los cuales los miembros de una sociedad pueden influir en el niño, ya que cuando se trata de fijar unos contextos u otros, las prácticas culturales van a tener un peso muy importante. Se señalan al menos cuatro factores de acuerdo con los cuales la cultura condiciona el desarrollo del niño: 1) la cultura organiza la presencia o ausencia de entornos problemáticos básicos a los que el niño ha de enfrentarse y que están incorporados a la prácticas culturales; 2 ) la frecuencia con que los niños realizan ciertas actividades, que se consideran básicas en un grupo social, está culturalmente organizada; 3) la cultura determina también las pautas de acuerdo con las cuales ciertos sucesos se presentan conjuntamente. 4) por último, la cultura regula el nivel de dificultad de las tareas que han de realizarse en un determinado contexto.

En suma esta aproximación está basada en dos principios. En primer lugar, existe una gran conexión entre los procesos de interacción social que se establecen en una cultura y los procesos psíquicos de sus miembros, ello es debido a que el funcionamiento psicológico del individuo emerge a través de la internalización de varios procesos que están presentes en la interacción social. b) El concepto de zona del desarrollo próximo proporciona un puente para que se establezcan las relaciones entre los niños y los adultos.

\section{EL PAPEL DEL ADULTO EN EL DESARROLLO COGNITIVO DEL NIÑO}

¿Cómo incide el adulto en el desarrollo cognitivo del niño? Esta es la cuestión esencial que se trata de responder. Ya hemos expuesto anteriormente, que el aprendizaje del niño tiene lugar, primero, en un plano social y solo después en un plano individual. El niño, al principio, resuelve los problemas en presencia de otros y gradualmente empieza a ejecutarlos por si mismo: primero el adulto controla y guía su actividad pero poco a poco ambos empiezan a compartir funciones en la resolución de la tareà; el niño comienza a llevar la iniciativa en la actividad, mientras que el adulto corrige y guía en los momentos de vacilación y duda; finalmente el niño toma plenamente el control y la responsabilidad de la tarea (Brown \& French 1979; Laboratory of Comparative Human Cognition 1983; Wertsch 1978). 
Debemos precisar, por lo tanto, cuál es la ayuda que el adulto puede proporcionar al niño. Han sido varios los autores que han señalado cómo la interacción niño-adulto es el precursor necesario para la adquisición de ciertas habilidades y destrezas básicas. Desde este punto de vista no se trata, simplemente, de favorecer el aprendizaje en situación concreta y en relación con un determinado contenido, es un proceso más complejo que permitirá a los niños no sólo aprender a resolver una tarea precisa, sino «aprender a aprender» (Brown, 1985). Esas habilidades de carácter general, que el niño adquiere en un contexto pero que podría generalizar a otros, resultan difíciles de determinar y ello es debido, sobre todo, a que no siempre se designan con los mismos términos y a que, además, diferentes procesos pueden tener funciones similares. En cualquier caso podemos señalar que, entre esas habilidades que el niño adquiere a través de su interacción con el adulto, las investigaciones más recientes señalan la importancia de capacidades estrechamente relacionadas con el uso del lenguaje, por ejemplo, la codificación de la tarea de acuerdo con diferentes niveles de complejidad o la autorregulación de la actividad mediante la palabra.

Veremos a continuación cómo el habla aparece, en el contexto de la psicología soviética, como uno de los pilares en los que se apoya el desarrollo cognitivo del niño y constituye una pieza esencial para explicar la influencia facilitadora que sobre él tiene el adulto. La palabra es el punto de apoyo desde el que los procesos intersubjetivos llegan a convertirse en intrasubjetivos. Si tenemos en cuenta algunos de los procesos que pueden intervenir en una tarea de aprendizaje o de solución de problemas, tal como se especifican en la figura 3, descubrimos fácilmente que el habla mantiene, al menos, esos dos tipos de relaciones, a los que acabamos de referirnos, con los procesos de conocimiento. En primer lugar, orienta la codificación de la información que recibe el sujeto a partir de la cual se define y se resuelve el problema; en segundo lugar se convierte en un importante instrumento en el control de la propia conducta haciendo más fácil su planificación.

\section{La importancia del lenguaje en el desarrollo del niño}

\section{La palabra como instrumento: sociogénesis del razonamiento}

La raíz de muchos trabajos actuales que examinaremos a continuación, dedicados a profundizar en el carácter funcional de la palabra y desde esta perspectiva en el papel de la interacción niño-adulto, está en la psicología soviética. Resulta difícil comprender sus aportaciones sin referirnos a la fuerte influencia de la obra de Marx sobre el pensamiento de Vygotsky. La psicología que se realiza en la Unión Soviética en torno a los años veinte está inmersa en el clima post-revolucionario y los planteamientos del materialismo histórico van a condicionarla profundamente. En este sentido la psicología de Vygotsky es no solo genética, como lo era la piagetiana, sino también social. Shina (1988) ha señalado en un trabajo muy reciente que sólo teniendo en cuenta este aspecto es posible comprender los análisis vygotskianos que considerar a la simbolización como instrumento del pensamiento y que proponen, en último extremo, una sociogénesis de razonamiento. El habla, en este contexto, se considera instrumento en un doble 


\section{4}

FIGURA 3

TAREA

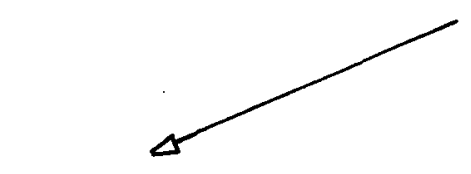

SUJETO

Codificación

Estructuración

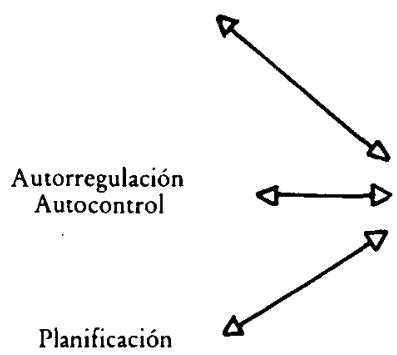

LENGUAJE

Habla Privada

4

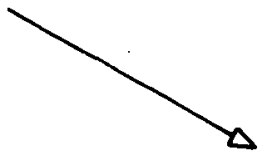

OBJETO

Información

RENDIMIENTO EN LA TAREA

Algunos procesos cognitivos implicitos en las situaciones de aprendizaje $y$ solución de problemas

sentido, por una parte permite la comunicación del niño con los demás y, por otra, un control de su conducta. Para comprender las relaciones que existen entre ambos aspectos es imprescindible acudir al concepto de «internalización» que tiene una profunda significación funcional. Como ya hemos señalado esa internalización es progresiva en cuanto que el lenguaje ha atravesado diversas fases, plenamente relacionadas con sus funciones, en las que se advierte con claridad la relación entre palabra y pensamiento. La evolución se produce, desde el habla pre-intelectual y pensamiento pre-verbal hasta el habla plenamente interiorizada cuyas funciones no son sólo sociales sino también intrapersonales. Existe una fase intermedia, entre las dos anteriores, la del habla egocéntrica o «habla privada" que tiene gran importancia en la etapa preescolar. Desde este punto de vista se considera (Luria, 1961; 1982; Vygotsky, 1962; 1978) que los niños pequeños usan el lenguaje no solo para comunicarse, sino también para guiar, planificar y controlar su propia actividad. Vygotsky observó que la palabra privada es emitida como una idea adicional a una actividad dada.

«El habla egocéntrica aparece cuando el niño intenta comprender la situación, encontrar una solución o planificar una actividad naciente. Los niños más mayores se comportan de una manera diferente: examinan el problema, piensan (lo cual está indicado por pausas más largas) y después encuentran una solución. Cuando se les pregunta en qué pensaban los niños responden en una línea si- 
milar al "pensamiento en voz alta" del preescolar. Asumimos que las mismas operaciones que el preescolar lleva a cabo en voz alta son relegadas a un habla interna y silenciosa en los escolares» (Vygotsky, 1986, p. 30).

Es bien sabido, por otra parte, que el tema de las funciones del habla egocéntrica fue uno de los principales puntos de desacuerdo entre Vygotsky y Piaget, para quien las palabras de los preescolares eran un signo de las dificultades infantiles en entender la perspectiva de otra persona. Piaget apoyaba así su postulado básico de que el pensamiento de los niños pequeños está centrado principalmente en su propio punto de vista. Vygotsky cuestionó estas conclusiones ya que, en su opinión, la palabra privada disminuye y desaparece con la edad no porque llegue a ser socializada, como sugirió Piaget, sino más bien porque va a constituir la palabra «intima» o pensamiento verbal.

\section{Interacción niño-adulto y habla privada: investigaciones recientes}

A partir de los años setenta se han realizado numerosas investigaciones sobre el habla privada. Si bién todos reconocen que tiene un efecto regulador sobre la actividad del niño, los resultados no siempre son coincidentes cuando se relaciona la presencia o ausencia del habla privada con la naturaleza de la tarea o el éxito o fracaso en la actividad. Citaremos algunos ejemplos. Bastantes estudios sobre el uso de la palabra privada en los niños han fracasado cuando tratan de encontrar relaciones positivas entre un uso espontáneo de esta y el éxito en la tarea (Yaeger, 1968; Zivin, 1972; Beaudichon, 1973; Dickie, 1973), no obstante Frauenglas y Díaz (1985) creen que esta carencia de correlación positiva no necesariamente contradice las afirmaciones teóricas de Vygotsky que había afirmado que el habla privada aumentaba en función de la dificultad de la tarea. La esperada, y usualmente observada, correlación entre palabra privada y fracaso en la tarea no contradice la idea de que tal palabra podría verdaderamente ser un instrumento usado por el niño para mejorar la ejecución. En esta línea se ha señalado que la palabra privada suele aparecer en momentos en que la tarea presenta especial dificultad (Goodman, 1981) o en función del grado de dificultad que pueden manifestar diferentes tareas (Berk, 1985, Fuson, 1985). No es sorprendente que estas dificultades incrementen la probabilidad del fracaso en la ejecución y, en esta línea, habrá que aceptar que la presencia de la palabra privada sería más frecuente en relación con el fracaso que con el éxito. En definitiva, parece posible admitir que las características de la tarea pueden enmascarar los efectos positivos de la palabra privada en su ejecución. En esta línea de trabajo Gaskill y Díaz (1987) llegan a la conclusión de que cuando se controla la dificultad de la tarea y se tiene en cuenta la mejora en el tiempo, y no la ejecución en un momento dado, hay una fuerte correlación entre la presencia de la palabra privada y la mejora en la tarea.

La cuestión que debemos plantear ahora es en qué medida la interacción del niño con el adulto favorecerá las funciones que Vygotsky asoció al habla privada, en cuanto que puede considerarse un instrumento de carácter simbólico. El niño preescolar no sólo actúa sino que también habla y ello le permite controlar las propias acciones, planificarlas e, incluso, transcender los estimulos que tiene ante sí considerando aspectos de la tarea que 
no están presentes en su reducido campo visual. Wertsh (1979) ha resumido con acierto como la interacción social puede generar procesos cognitivos, muy similares a los que hace surgir el habla privada, que favorecen el aprendizaje y la solución de problemas:

«El niño es incapaz de formular y ejecutar muchas acciones independientemente porque no puede representarse una meta que no está directamente sugerida por el entorno; esto es, el niño sólo puede realizar independientemente aquellas acciones dirigidas a una meta que no requiere una representación descontextualizada. Si el niño de temprana edad lleva a cabo acciones dirigidas a metas que requieren esta forma abstracta de representación, sus intentos tendrán que ser mediatizados y regulados por un adulto capaz de formar esa representación" (Wertsch, 1979, p. 89).

El texto alude a que los niños comienzan a regular su actividad cuando son capaces de utilizar por sí mismos, en un plano intrapsíquico, aquellos instrumentos de carácter simbólico que usaban mediatizados por los adultos. Entre estos instrumentos el principal es «el habla directiva».

Revisaremos algunos trabajos que analizan la relación del niño con el adulto considerando, desde el punto de vista que acabamos de señalar, que esta situaçión favorece la internalización de instrumentos simbólicos que tienen un importante papel en el control de la conducta. Con el fin de introducir una cierta sistematización en un campo de estudio enormemente complejo diferenciaremos dos tipos de trabajos a cuya temática ya hemos aludido anteriormente. En primer lugar, aquellos que insisten en que esa interacción juega un importante papel en el paso de la regulación de la propia conducta a partir de los otros a la autorregulación, entendida ésta como el éxito en la resolución de problemas que se ejecutan independientemente. Nos detendremos especialmente en los que se han dedicado a estudiar la planificación; por otra parte, los que consideran que la relación con el adulto facilita el acceso del niño a la información necesaria para resolver la tarea, desde este punto de vista puede aceptarse que los adultos facilitan al niño sus procesos de codificación.

\section{¿Cómo favorece el adulto la autorregulación infantil?}

Ann Brown (1987) relaciona la autorregulación con la metacognición, considerando ésta como un conjunto de procesos que permiten al niño dirigir la atención a su propio funcionamiento cognitivo. Se interesa sobre todo por sus dimensiones funcionales ya que considera que facilita el control que el niño puede ejercer sobre su propia conducta, en este sentido la autorregulación está en estrecha relación con procesos como la planificación, el control ejecutivo, la revisión y corrección de los errores, etc. En otro lugar hemos revisado con mayor detalle este proceso (García Madruga y Lacasa, 1989). Diferentes investigaciones han señalado cómo el contexto (Brown y Reeve, 1987) y, sobre todo, determinadas situaciones de interacción niño-adulto facilitan su desarrollo. Desde este punto de vista conviene resaltar que aunque, de acuerdo con algunos estudios recientes, la metacognición parece ser una de las habilidades cognitivas que se benefician de la participación social en mayor medida (McLane, 1987; Hartup, 1985), otras investigaciones (Kontos, 1983) no han obtenido datos totalmente con- 
cluyentes en este punto. Nos acercaremos al problema citando como ejemplo algunos trabajos concretos.

\section{El control de la conducta infantil: la metacognición}

Wertsch y. Hickmann (1987) analizan el proceso de interacción niñoadulto, en situaciones de solución de problemas, para determinar de qué modo los adultos regulan las conductas de los niños. En el estudio participan nueve díadas de madres con sus hijos. Las edades de los niños, que asistian a un preescolar, estaban comprendidas entre 2,6 y 4,6 años. La tarea consistía en la realización de un puzzle por parte de niños, de acuerdo con un modelo, con ayuda de la madre. Los resultados se analizaron a partir de una codificación de las interacciones de ambos, segmentadas en «episodios", éstos incluían todas las conductas implicadas en la colocación de una pieza; aunque la mayoría de las veces la colocación era correcta, los niños realizaron en ocasiones colocaciones incorrectas que la madre no intentó corregir. Los episodios se clasificaron teniendo en cuenta la dificultad de cada una de las piezas para ser situada correctamente. Los autores presentan en este trabajo análisis microgenéticos de dos díadas. En la primera, que corresponde a la interacción de un niño de 4,6 años con su madre se advierte un cambio en la distribución de las estrategias de control a través de los sucesivos episodios; existe un paso de la autorregulación a través de la madre a la autorregulación por parte del niño; dicho control se observa en que aquella, al principio de la sesión, dirige la atención del niño al modelo - general o específico-, selecciona las piezas, las sitúa, etc., pero progresivamente es el niño quien realiza la actividad y toma la iniciativa. En definitiva, se asiste a la génesis de conductas de autocontrol que suponen una revisión de la propia actividad en curso. En la segunda pareja, en la que el niño tiene 3,6 años, es la madre la que, durante todos los episodios, controla la actividad del niño. Los resultados del trabajo mostraron que la regulación de la madre, en forma de miradas, indicaciones, indirectas, etc., aumentaba en función de la edad del niño; es decir, existía un mayor control de la conducta por medio de los otros cuando los niños eran más pequeños. A modo de conclusión puede afirmarse que dicho control sobre la conducta infantil se produce porque los adultos proporcionan al niño un amplio repertorio de regulaciones tanto verbales como no verbales.

\section{¿Es la interacción niño-adulto el «único» factor en la génesis de la metacognición?}

Una investigación que Susan Kontos (1983) realizó hace ya algunos años había llegado a conclusiones parcialmente diferentes a las de la investigación de Werstch y Hickman (1987). En su trabajo presenta dos experimentos cuyo fin era analizar el impacto en los procesos de metacognición infantil de la interacción del niño preescolar con el adulto. Consideraba que dicha interacción mejoraría el rendimiento de los niños en la tarea. Seleccionó una tarea difícil, pero que podía lograrse con la ayuda de un adulto, que consistía en la realización de un puzzle. En el primer estudio participan 39 niños, cuya media de edad era de 4,1 años y sus madres. El principal objetivo era proporcionar una descripción detallada del entorno que fa- 
vorece la metacognición, se analizó la dirección verbal que la madre proporciona al niño mientras resuelve el problema y su incidencia en la actividad del niño. La madre era instruida previamente en cómo resolver el puzzle y en cómo podía ayudar verbalmente al niño; no existía, sin embargo, ninguna imposición y ella podía elegir el método de ayuda que considerara más conveniente. Tampoco se fijaba un tiempo límite para la interacción de la madre con su hijo. Las sesiones estaban divididas en tres fases, en la primera y la tercera el niño interactuaba con el experimentador y en ambas se determinaba, por una parte, el número de piezas que el niño había colocado correctamente y, por otra, el conocimiento que el niño tenía de las estrategias que había utilizado, para ello era interrogado por el experimentador una vez que había realizado la tarea. En la segunda de las fases el niño resolvía el problema con la madre. Los resultados se analizaron a partir de una categorización de las conductas de ambos y este aspecto es, en nuestra opinión, una de las aportaciones más importantes del estudio. Las categorías para analizar las verbalizaciones de la madre a partir de su contenido metacognitivo incluían, por ejemplo, referencia a la meta, conocimiento de aspectos perceptivos o conceptuales que facilitaban la tarea, división de la tarea con el fin de que el niño pudiera ir dando cada uno. de los pasos separadamente, etc. Las categorías se ordenaron, además, en dos grupos: las que se referían a la tarea y las que aludían a la estrategia del niño. Los resultados mostraron que en las conductas verbales de la madre existe un gran número de verbalizaciones con contenido metacognitivo $(69 \%)$ en relación con el número total de verbalizaciones emitidas. Dichas verbalizaciones variaban en función de la edad del niño, tanto en la cantidad como en su naturaleza; eran más abundantes en las madres de niños pequeños $\mathrm{y}$, en este caso, también más simples, por ejemplo, no se verbalizaba la estrategia total sino el movimiento de una sola pieza. Las correlaciones entre estas verbalizaciones de la madre y el logro en la tarea de sus hijos fueron bajas aunque algunas de ellas significativas.

En el segundo estudio que presenta Susan Kontos (1983) se pretenden ampliar las conclusiones del primero. Entre sus objetivos quizá era el más importante contrastar las diferencias existentes entre un grupo de niños que recibían asistencia metacognitiva y otros que estando inmersos en una situación social similar, en este caso la interacción con el padre, no tenían ese tipo de asistencia. En este grupo los padres no habían conocido la tarea previamente y se limitaban a animar a los niños cuando colocaban las fichas en una posición correcta. En este caso, a diferencia del anterior, no existían correlaciones entre el logro en la tarea y las afirmaciones metacognitivas de los padres de los niños del grupo experimental. Además, los niños del grupo de control, que no recibió ayuda metacognitiva, mejoraron su rendimiento de un modo bastante similar a los niños del grupo experimental. Kontos, a partir de estos dos estudios, llegó a la conclusión de que un mejor rendimiento en la tarea puede ser debido tanto a la interacción con el adulto que favorece la metacognición como a los efectos de la repetición. Esto le hace pensar, por otra parte, que aunque la interacción niñoadulto fomenta la adquisición de ciertas destrezas no se puede decir que es un precursor necesario para el desarrollo de las habilidades metacognitivas. En su opinión nuevos estudios habrán de contrastar el papel que tienen en el origen de la metacognición el plano interpsíquico, la interacción niño- 
adulto y el plano intrapsíquico, resolver un problema individualmente. Ninguno de estos dos niveles debe ser olvidado.

\section{El papel de la interacción niño-adulto en conductas de planificación}

\section{¿Qué significa "planificar»?}

Cuando Ann Brown (1987), analiza las raíces teóricas de la metacognición, entendida como la capacidad del sujeto de regular sus procesos de conocimiento, la asocia, entre otros, a un mecanismo preciso: la planificación. Actualmente este tema es objeto de múltiples investigaciones que se sitúan en dos perspectivas teóricas: la psicología computacional (Hayes-Roth y Hayes-Roth, 1979; Willensky, 1983) y la que se inspira en los planteamientos más clásicos del pensamiento soviético (Rogoff, Gauvain y Gadner, 1987). Nos ocuparemos de estos últimos debido a la importancia que en ellos adquiere el papel del contexto y, más concretamente, la interacción niño-adulto como situación adecuada para favorecer las conductas de planificación.

Con el término planificación suele aludirse en la literatura psicológica a diversas facetas del funcionamiento cognitivo, de ahí que estemos ante un término un tanto difuso y vago. Rogoff, Gauvain y Gardner (1987) definen a la planificación como la organización deliberada de una secuencia de acciones orientadas hacia el logro de un objetivo específico. Un plan es un programa que puede hacer referencia a los objetos, al lugar y al momento en relación con los que una acción va a tener lugar. Scholnick y Friedman (1987) se refieren a diversos aspectos que han interesado a los psicólogos del desarrollo en relación con la planificación, todos ellos son un buen ejemplo de los procesos que intervienen en la regulación de la conducta: la representación de las metas hacia las que se orienta la actividad en cuanto que facilita el autocontrol, la adquisición de estrategias que facilitan la coordinación de diferentes habilidades implícitas en la consecución de una meta, la metacognición, etc. Todos estos aspectos están implícitos en el planteamiento del tema que está presente en la psicología soviética. Luria (1979), por ejemplo, señala que los orígenes de la planificación deben atribuirse a la habilidad verbal que fomenta tanto el desarrollo de la representación como el desarrollo del autocontrol.

\section{El papel del adulto en la planificación infantil: algunos trabajos recientes}

Con el fin de analizar cómo la interacción con el adulto puede favorecer la planificación, revisaremos con detalle algunos de los trabajos más recientes de Barbara Rogoff y sus colaboradores que, en la actualidad, trabajan en este problema. Una de sus preocupaciones más importantes es analizar las relaciones entre el contexto y los procesos de planificación. Sus trabajos abordan el tema desde un doble punto de vista y aludiendo siempre al contexto. Por una parte, se refieren a la necesidad de adaptación a las circunstancias en las que va a desarrollarse la actividad que se proyecta y que está implícita en un proceso de planificación; el contexto, desde este punto de vista, representa las exigencias que plantea el entorno y a las que habrá de acomodarse el niño. Pero, por otra parte, el contexto puede con- 
vertirse también en un elemento facilitador de la planificación, sobre todo cuando se considera la interacción del niño con planificadores más expertos, por ejemplo, los adultos. De estas dos cuestiones nos ocuparemos brevemente a continuación.

Veremos, en primer lugar, cuáles son las diferencias entre planificadores expertos y novatos, en función de cómo ambos se adaptan a las circunstancias. Existen dos criterios que permiten diferenciar entre las estrategias de planificación, aun con riesgo de simplificar excesivamente el problema. El primero se refiere a la necesidad de discriminar qué contextos son los más apropiados para planificar una determinada actividad, en este sentido aparece que los niños comienzan desarrollando sus competencias en contextos específicos y sólo los planificadores expertos son capaces de generalizarlas a nuevos contextos. Estas habilidades dependen de cuándo y cómo se hayan adquirido y el niño habrá de aprender cómo ajustarse a cada situación en función de las circunstancias. Todo ello supone, en último extremo, establecer relaciones adecuadas entre la meta que se pretende lograr y los medios que han de utilizarse en cada caso (Rogoff, Gauvain y Gadner, 1987). Un segundo criterio permite diferenciar las estrategias de planificación se refiere a la distinción entre la planificación por anticipado y la que acompaña la ejecución de la tarea. En líneas generales, los planificadores expertos difieren de los novatos, en que los primeros formulan una estrategia general y un plan provisional al principio que va guiando la actividad y que se reformulan en el curso de la acción; además, hacen más explícitas sus decisiones y usan con más eficacia la información disponible. Sin embargo, en los planificadores novatos las decisiones son inmediatas y no tienen una estrategia general para la solución de la tarea (Radziszewska y Rogoff, 1988).

Desde esta perspectiva se ha comprobado que las habilidades de planificación se desarrollan con la edad. Gardner y Rogoff (1986) han mostrado, analizando como los niños resuelven un problema relacionado con un laberinto, que los niños mayores (cuyas edades oscilaban entre los 7 y 9,6 años) utilizaban distintas estrategias de planificación en función de las condiciones del problema; por el contrario, los niños más pequeños (sus edades se distribuían entre los 4,6 y 7 años) no eran capaces de tener en cuenta el contexto de la tarea. Los autores interpretan estos datos como algo que puede ser debido, al menos en parte, a las dificultades de los pequeños de coordinar los medios y el objetivo en la resolución de problemas complejos.

Hemos señalado ya, anteriormente, que el contexto puede facilitar la planificación, sobre todo cuando se considera la interacción del niño con planificadores más expertos (Cole, Hood y McDermott, 1978; Rogoff, 1982; Rogoff, Gauvain y Gardner, 1987). Se ha comprobado, por ejemplo, que los niños aprenden a planificar mejor si comparten la responsabilidad de la tarea con lo adultos o compañeros (Gauvain y Rogoff, 1986). Por otra parte los individuos que son expertos en una actividad de planificación pueden ayudar a los novatos estructurando el problema y guiando al niño en su solución (Rogoff, 1986; Rogoff, Malkin y Gilbridge 1984; Saxe, Gearhart y Guberman, 1984), en este sentido, por ejemplo, los adultos presentan la tarea señalando un conjunto de submetas que el niño va logrando progresivamente acercándose a la meta principal de una manera gradual. 


\section{El papel de la interacción niño-adulto en la codificación y estructuración de la tarea}

En las páginas anteriores se ha señalado ya repetidamente como la ayuda que el adulto proporciona al niño puede ser múltiple y como dicha ayuda se comprende mejor si se analiza en relación con la internalización progresiva, por parte del niño, de formas de conducta socialmente adquiridas entre las que el lenguaje tiene un lugar fundamental. La adquisición del habla y la capacidad de utilizarla como un instrumento simbólico está mediatizada, sin lugar a dudas, por la interacción del niño con el adulto. Entre dichas funciones se destacaban especialmente tres, la primera y la segunda relacionadas con la capacidad de autocontrol y planificación, la tercera con la codificación y estructuración de la información necesaria para resolver una tarea. De este útlimo aspecto nos ocuparemos a continuación.

Los estudios que han analizado en profundidad las situaciones de enseñanza-aprendizaje en las que intervienen niños o adultos como enseñantes, suelen referirse a las diferentes estrategias de acuerdo con los cuales ambos se acercan a la información que debe transmitirse al que aprende y que es necesaria para resolver una tarea determinada. Dichas estrategias podrían estar condicionadas, al menos en alguna medida, por los procesos de codificación que debe realizar el que enseña; estos procesos, por otra parte, están íntimamente relacionados con la capacidad de utilizar un sistema simbólico más o menos complejo.

Nos referimos brevemente, citando algunos estudios empíricos, a los diferentes efectos que se han observado, considerando que no solo los audltos sino también otros niños pueden ser enseñantes. En los años setenta, por ejemplo, algunos investigadores habrían asumido la idea de que los niños pueden ser profesores muy efectivos (Allen, 1976; Cicirelli, 1976; Steward y Steward, 1974). Entre las razones que se aducian para mantener esta posición se decía que cuando un niño enseña a otro ambos compartían el mismo nivel de lenguaje, y de aquí que, en algunas ocasiones, pudieran enseñar mejor que los adultos.

Rogoff y su grupo de investigación han realizado numerosos trabajos sobre esta cuestión (Rogoff, 1981; Ellis y Rogoff, 1982; Rogoff, 1984; Rogoff, Ellis y Gadner, 1984) que no parecen haber llegado a los mismos resultados. Comentaremos brevemente uno de sus estudios que sirve como ejemplo para ilustrar esas diferencias a las que nos venimos refiriendo. Ellis y Rogoff (1986) realizaron unos estudios que sirve como ejemplo para ilustrar esas diferencias a las que nos venimos refiriendo. Ellis y Rogoff (1986) realizaron una investigación cuyo objetivo era analizar la eficacia de las estrategias instruccionales que niños y adultos introducen en un proceso de enseñanza aprendizaje. En el estudio participan 32 parejas, en 16 de ellas los «maestros» eran niños entre 8-9 años y en los otros 16 "maestros» eran mujeres adultas. La edad de los niños que debían aprender era de 6-7 años. La tarea que se trataba de enseñar era una clasificación de dos tipos de elementos, unos relacionados con los objetos que habitualmente los niños encuentran en la cocina de su casa, los otros, representados en fotografías, se referían a objetos muy variados. Tanto los que enseñaban como los que aprendían fueron informados de la importancia de conocer la organización de los elementos, ya que una vez que los niños hubieran aprendido la tarea 
deberían realizarla ellos solos. No podemos exponer ahora en detalle todos los resultados del trabajo pero es importante aludir a las diferencias encontradas entre las estrategias de los «maestros» niños o adultos. Estos últimos, por ejemplo, ayudan a los niños que aprendan a ir más allá de las metas inmediatas, por ejemplo no se limitan a colocar únicamente un elemento en su posición correcta, por el contrario les facilitan la comprensión de la categoría de clasificación lo que hará más fácil clasificar todos los elementos. También los adultos transmiten mayor cantidad de información y parecen conocer mejor el nivel de comprensión del niño al que ayudan. En definitiva, este trabajo muestra que los adultos como enseñantes pueden facilitar mejor al que aprende una comprensión de la estructura de al tarea que ayudará la solución del problema.

\section{CONCLUSIONES}

No resulta fácil resumir en pocas líneas cómo la interacción del niño con el adulto facilita el desarrollo cognitivo. En este trabajo hemos querido mostrar que existen múltiples vías para abordar el problema y que éstas dependen, en último extremo, de la perspectiva teórica en la que se sitúa el investigador, Mas concretamente, hemos mostrado como el hecho de adoptar un determinado modelo para representar «el contexto", una de cuyas dimensiones es la interacción niño-adulto, implica aceptar una línea de investigación que condiciona no solo el tipo de cuestiones que se plantean sino también los métodos propuestos para resolverlas y las explicaciones de los hechos. Hemos considerado, a título de ejemplo, distintas aproximaciones teóricas desde las que es posible plantear el tema de las relaciones entre el contexto y los procesos cognitivos. Para quienes se inspiran en la biología y que consideran el conflicto como un mecanismo esencial para explicar el desarrollo, uno de sus representantes más claros sería Piaget, el papel del adulto es similar al de otros agentes de carácter social o físico que plantean dificultades que el niño habrá de superar en su proceso de adaptación al medio. Desde otra perspectiva, pero también insistiendo en esa misma necesidad de adaptación, los psicólogos próximos a la etología analizan la relación del niño con el adulto en situaciones naturales, en nuestra opinión estos trabajos buscan más describir el entorno que dar explicaciones acerca de su incidencia en el desarrollo. Hemos mostrado también cómo los diferentes niveles de análisis que se proponen en el modelo ecológico de Bronfenbrenner permiten diversos enfoques de la interacción niño-adulto y analizan, sobre todo, la influencia que el adulto ejerce sobre el niño teniendo cuenta, a su vez, las condiciones del entorno que condicionan la conducta del adulto. Por último hemos revisado los trabajos inspirados en la psicología soviética, con una fuerte influencia de la antropología, que se han visto obligados a dar cuenta de un hecho importante: la sociedad y la cultura aportan a sus miembros diferentes tipos de experiencia que condicionan su conocimiento. Numerosos trabajos se han dedicado a analizar la influencia del adulto sobre el desarrollo del niño asumiendo, al menos, dos de las aportaciones más significativas de Vygotsky, tematizadas en relación con dos conceptos: «zona del desarrollo próximo» e «internalización». Desde este punto de vista hemos señalado cómo la influencia del adulto esta 
estrechamente relacionada con el uso de la lengua; la palabra, que se adquiere progresivamente en la interacción con el adulto, se convierte en un elemento capaz de regular la conducta y, además, permite una codificación cada vez más compleja de la realidad.

\section{Referencias}

ALLEN, V. L. (1976). Children heping children: psychological process in tutoring. En J. R. Levin y V. L. Allen (Ed.): Cognitive learning in children: theories and strategies. Nueva York: Academic Press.

Bandura, A. (1977). Self éfficacy: Toward a unifying theory of behavioral. Psychological Review, 84 191-215.

BEAUDICHON, J. (1973). Nature and instrumental function of private speech in problem solving situations. Merrill-Palmer Quarterly, 19, 117-135.

BERCK, L. E. (1985). Why children talk to themselves. Young Children, July, 46-52. BowlBY, J. (1969). Attachment and Loss. Vol. 1. Attachment. Nueva York: Basic Books.

BRONFENBRENNER, U. (1974). Developmental research, public politicy, and the ecology of childhood. Child Development, 45, 1-5.

BRONFENBRENNER, U. (1979). The Ecology of Human Development: Experiments by nature and design. Cambridge, Mass.: Harvard University Press.

BRONFENBRENNER, U.; ALVAREZ, V., y HENDERSON, C. (1982). Working and watching: maternal employment status and parents perceptions of their three-year-old children. Ithaca, N. Y.: Department of Human Development and Familie Studies, Cornell University.

BRONFENBRENNER, U., y CROUTER, A. (1983). The evolution of environmental models in developmental research. En P. H. Mussen (Ed.): Handbook of Child Psychology (4th ed.) History, Theory, and Methods. Vol. 1. Nueva York: Wiley.

BROWN, A. L. (1985). Mental orthopedics, the training of cognitive skills:An interview with Alfred Binet. En S. F. Chipman, J. W. Segal y R. Glaser (Ed.): Thinking and Learning Skills. Research and Open Questions. Vol. 2. Hillsdale, N. J.: LEA.

BROWN, A. L. (1987). Metacognition, executive control, self-regulation and other more mysterious mechanisms. En F. E. Weinert y H. Kluwe (Ed.): Metacognition, motivation and understanding. Hillsdale, N. J.: LEA.

BROWN, A. L., y REEVE, R. A. (1987). Bandwidths of competence: The role of supportive context in learning and development. En L. S. Lyben (Ed.): Development $\&$ Learning: Conflict or Congruence. Hillsdale, N. J.: LEA.

Brown, A. L., y FRENCH, L. A. (1979). The zone of potential development: implications for intelligence testing in the year 2000. Intelligence, 3, 255-257.

CHARLESWORTH, W.R. (1978). Ethology: understanding the other half of intelligence. Social Science Information, 7, 231-277.

CHARLESWORTH, W. R. (1983). An ethological approach to cognitive development. En Ch. J. Brainerd (Ed.): Recent Advances in Cognitive Developmental Theory. Progress in Cognitive Developmental Research. Nueva York: Springer Verlag.

CiCIRELLI, V. G. (1976). Sibling teach sibling. En V. L., Allen (Ed.): Children as teachers: theory and research on tutoring. Nueva York: Academic Press.

Cochran, M. M., y RoBInson, J. (1982). Day care and sex differences. En S. Kilmer (Ed.): Advances in early education and day care. Vol. 3. Greenwich, Coon.: J. A. I. Press.

COLE, M. (1975). An etnographic psychology of cognition. In R. W. Brislin; S. Bochner, y W. J. Lonner (Ed.): Cross-Cultural Perspectives on Learning. Nueva York: Halsted Press.

COLE, M.; HOOD, L., y MCDERMOTT, R. P. (1978). Ecological niche picking: Ecological invalidity as an axiom of experimental cognitive psychology. Umpublished manuscript, University of California, San Diego, y The Rockefeller University.

COLE, M., y GRIFFIN, P. (1980). Cultural amplifiers reconsidered. En D. Olson (Ed.): Social Foundations of languague and thought. Nueva York: Norton.

Del Río, F., y Alvarez, A. (1985). La influencia del entorno en la educación. Infancia y aprendizaje, 29, 3-33.

DELOACHE, J. S. (1980). Naturalistic studies of memory for object location in very young children. New directions for Child development, 10, 17-32.

DiCKIE, J. R. (1973). Private speech: The effect opresence of others, task and intrapersonal variables. Dissertation Abstracts International, 34, 1292.

DOISE, W. (1983). Apprentissage, psychologie génétique et psychologie social, une transformation de paradigmes. Archives de Psychology, 51, 17-22.

DoIsE, W. (1985). Psychology sociale et constructivisme cognitif. Archives de psychologie, 53, $127-140$. 
DoIse, W., y MugnY, G. (1981). Le développement social de l'intelligence. París: InterEditions.

ELLIS, S., y ROGOFF, B. (1982). The strategies and efficacy of child versus adult teachers. Child Development, 53, 730-735.

ElLIS, S., y ROGOFF, B. (1986). Problem solving in children's management of instruction. En E. C. Mueller y C. R. Cooper (Ed.): Process and outcome in peer relationship. Orlando: Academic Press.

ETIENNE, A. S. (1984). The meaning of object permanence at different zoological levels. $\mathrm{Hu}$ man Development, 27, 309-320.

Frauenglas, M. H., y Díaz, R. M. (1985). Self-Regulatory functions of children's private speech: A critical analysis of recent challenges to Vygotsky's theory. Developmental Psychology, 21, 357-364.

FusoN, K. C. (1985). The development of self-regulating aspects of speech: A review. En G. Zivin (Ed.): The Development of Self-Regulation Through Private Speech. Nueva York: John Wiley \& Sons.

GADNER, W., y ROGOFF, B. (1986). The development of flexibility in children's improvisational and advance planning (documento no publicado).

Garcia Madrugada, J., y LACASA, P. (1989). Procesos cognitivos básicos. En J. Palacios, A. Marchesi y C. Coll (Ed.): Psicologia Evolutiva. Barcelona: Paidós.

GASKILL, M. M., y DIAZ, R. (1987). The relation between private speech and cognitive performance (documento no publicado).

GAUVAIN, M., y RogOFF, B. (1986). (en prensa). Collaborative problem solving and children's planning skills.

Gelman, R. (1978). Cognitive development. Annual Review of Psychology, 29, 297-232.

GOODMAN, S. H. (1981). The integration of verbal and motor behavior in preschool children. Child Development, 52, 280-289.

GunNarson, L. (1978). Children in day care and family care in Sweden: A follow-up. Gothenburg, Sweden: University of Gothenburg.

HARTUP, W. (1985). Relationship and their significance in cognitive development. En R. Hinde y A. Perret Clermont (Ed.): Relationships and cognitive development. Oxford: Oxford University Press.

HAYES-ROTH, B., y HAYES-Roth, F. (1979). A cognitive model of planning. Cognitive Science, 3, 275-310.

Hichman, M. (Ed.) (1987). Social and Functional Approaches to Language and Thought. Orlando: Academic Press.

HUNDE, R. A. (1983). Ethology and child development. En P. H. Mussen (Ed.): Handbook of Child Psychology (4th ed.) Vol. 2. M. H. Marshall y J. J. Campos (Ed.): Infancy and Developmental Psychobiology. Nueva York: Wiley.

HOFFMAN, L. W. (1980). The effects of the maternal employment on the academic attitudes and performance of school-aged children. School Psychology Review, 9, 319-335.

KINGMA, J. (1984). The influence of task variations in seriation research: adoling irrelevant cues to stimulus material. The Journal of Genetic Psychology, 144, 241-253.

KONTOS, J. (1983). Adult-Child interaction and the origins of metacognition. Journal of Educational Psychology, 77, 43-54.

Laboratory of Comparative Human Cognition. (1983). Culture and cognitive development. En P. H. Mussen (Ed.): Handbook of child Psychology. Vol. 1. W. Kessen (Ed.): History, theory, and methods. Nueva York: Wiley.

LACASA, P. (1984). Equilibrio y equilibración en la epistemología genética. Estudios de Psicologia, 93-115.

LEONTIEV, A. (1981). The problem of activity in psychology. En J. Wertsch (Ed.) (1981). The concept of activity in soviet psychology. Nueva York: M. E. Sharpe, Inc. Publisher.

LEONTIEV, A. (1983). El desarrollo del psiquismo. Madrid: Akal Universitaria.

LóPEZ, F. (1983). Etología. En A. Marchesi, M. Carretero y J. Palacios (Ed.): Psicología evolutiva 1. Teorias y métodos. Madrid: Alianza.

LORENZ, K. (1981). The Foundations of Ethology. Nueva York: Springer Verlag.

LURIA, A. R. (1961). The role of speech in the regulation of normal and abnormal behavior. Oxford: Pergamon Press.

LURIA, A. R. (1982). Language and cognition. Nueva York: John Wiley \& Sons.

LURIA, A. R. (1984). Conciencia y lenguaje. Madrid: Visor Libros.

MCDERMOTT, R. P., y ROTH, D. R. (1978). The social organization of behavior: Interactional approache. Annual Review of Antropology, 7, 321-345.

MCLANE, J. B. (1987). Interaction, context and the zone of proximal development. En M. Hichman (Ed.) (1987). Social and Functional Approaches to Language and Thought. Orlando: Academic Press.

MURRAY, F. B. (1983). Learning and development through social interaction and conflict: A challenge to social learning theory. En L. S. Lieben (Ed.): Piaget and the foundations of knowledge. Hillsdale, LEA. 
Perret Clermont, A. N. (1984). La construcción de la inteligencia en la interacción social. Madrid: Aprendizaje Visor.

PIAGET, J. (1967). Biologie et connaissance. París: Gallimard.

RADZISZEwSKA, B., y ROGOFF, B. (1988). Influence of adult and peer collaborators on children's planning skills. Developmental Psychology, en prensa.

ROBERT, M., y ChARBONNEAU, C. (1977a). Extintion of liquid conservation by observation: Effects of models, age and presence. Child Development, 48, 648-652.

Robert, M., y ChARBONNEAU, C. (1977b). Extintion of liquid conservation by observation: Three indicators of its artificiality. Child Development, 49, 194-200.

ROBERT, M. (1983). Observational learning of conservation. The independence from social influence. British Journal of Psychology, 74, 1-10.

ROGOFF,B. (1981). Adults and peers as agents of socialization: A highland guatemalan profile. Ethos, spring, 18-36.

RogOFF, b. (1982). Integrating context and cognitive development. En M. E. Lamb y A. L. Brown (Ed.): Advances in Developmental Psychology. Vol. 2. Hillsdale, N. J.: LEA.

RoGOFF, B. (1984). Adult guidance of cognitive development. En B. Rogoff y J. Lave (Ed.): Everyday Cognition: Its Development in Social Context. Cambridge, M.: Harvard University Press.

RogOFF, B. (1986). Adult assistance of children's learning. En T. E. Raphael (Ed.): The Context of School-based literacy. Nueva York: Random House.

ROGOFF, B. (1987). The joint socialization of development by young children and adults. En M. Lewis y S. Feinman (Ed.): Social influences and behavior. Nueva York: Plenum.

ROGOFF, B.; ELLIS, S., y GADNER, M. (1984). Ajustment of adult-child instruction according to child's age and task. Developmental Psychology, 20, 192-199.

Rogoff, B.; GAUVAIN, M., y GARDNER, W. (1987). Children's adjustment of plans to circunstances. En S. L. Friedman, E. K. Scholnick y R. R. Cocking (Ed.): Blueprints for Thinking. The role of Planning in Cognitive Development. Cambridge: Cambridge University Press.

RoGOFF, B.; MALKIN, C., y GILBRIDGE, K. (1984). Interaction with babies as guidance in development. En B. Rogoff $y \mathrm{~J}$. Wertsch (Ed.): Children's learning in the azone of proximal development.. San Francisco: Jossey Bass.

RUSSELL, J. (1981). Why sociocognitive conflict may be imposible: The status of egocentric errors in the dyadic performance of a spend task. Educational Psychology, 1, 159-160.

RuSSELL, J. (1982). Cognitive conflicts transmission and justification: Conservation attainment through dyadic interaction. The Journal of genetic Pchology, 140, 283-297.

SAMEROFF, A. J. (1982). Development an the dialectic: the need for a systems approach. En W. A. Collins (Ed.): Minnea Symposium on Child Psychology. Vol. 15. Hillsdale, N. J.: LEA.

SAMEROFF, A. J. (1983). Developmental systems: contexts and evolution. En P. H. Mussen (Ed.): Handbook of Child Psychology (4th ed.) History, Theory, and Methods. Vol. 1. Nueva York: Wiley.

SAXE, G. B.; GeARHART, M., y GUBERMAN, S. R. (1984). The social organization of early y number of development. En B. Rogoff y J. Wertsch (Ed.): Children's learning in the azone of proximal development». San Francisco: Jossey Bass.

SCHOLNICK, E. K., y FRIEDMAN, S. L. (1987). The planning construct in the psychological literature. En S. L. Friedman, E. K. Scholnick y R. R. Cocking (Eds.): Blueprints for thinking. The role of planning cognitive development. Cambridge: Cambridge University Press.

SINHA, C. (1988). Language and Representation. A Socio-Naturalistic Approach to Human Development. Nueva York: Harvester-Weatsheaf.

SMITH, M. B. (1968). School and home: Focus on achievement. En A. H. Passow (Ed.): Developing programs for the educacional disadvantaged. Nueva York: Teachers College Press.

STEWARD, M., y STEWARD,D. (1974). Parents and siblings as teachers. En J. Mash, L. C. Handy y L. A. Hamerlych (Ed.): Behavior modification approaches to parenting. Nueva York: Brunner/Mazel.

TINBERGEN, N. (1972). The animal and his world. Londres: G. Allen and Unwin.

VyGOTSKy, L. S. (1978). Mind in Society. The Development of Higher Psychological Processes. Cambridge, M.: Harvard University Press.

VygOTSKY, L. S. (1986). Thought and Language. Cambridge, M.: The MIT Press.

WADDINGTON, C. H. (1976). Las ideas básicas de la biología. En C. H. Waddington y otros. Hacia una biología teórica. Madrid: Alianza Universidad.

WERTSCH, J. (1978). Adult-Child interaction and the roots of metacognition. Quarterly Newsletter of the Institute for Comparative Human Development, 2, 15-18.

WERTSCH, J. (1979). The regulation of human action and the given new organization of private speech. En G. Zivin (Ed.): The Development of Slef-Regulation Through Private Speech. Nueva York: John Wiley \& Sons.

WERTSCH, J. (Ed.) (1981). The concept of activity in soviet psychology. Nueva York: M. E. Sharpe, Inc. Publisher. 
WERTSCH, J. (Ed.) (1985). Culture, communication, and cognition: Vygotskian perctives. Cambridge: Cambridge University Press.

WertsCh, J., y HickMaN, M. (1987). Problem solving in social interaction: A microgenetic analysis. En M. Hickman (Ed.): Social and Fuctional Approaches to Language and Thought. Orlando: Academic Press.

WILENSKY, R. (1983). Planning and Understanding. A Computational Approach to Human Reasoning. Massachusetts: Addison-Wesley Publishing Company.

YAEGER, J. A. (1968). Self directing speech and puzzle-solving in children. Unpublished doctoral dissertation, University of Chicago.

Zimmerman, B. J. (1983). Social learning theory: A contextualist account of cognitive functioning. En CH. J. Brainerd (Ed.): Recent advances in cognitive-developmental theory. Progress in cognitive development research. Nueva York: John Wiley \& Sons.

ZIvIN, G. L. (1972). Functions of private speech during problem solving in preschool children. Dissertation Abstracts International, 33, 1834.

\section{Extended summary}

The approach proposed in this paper aims for an integration of context and cognitive development. Children adapt to the constrains and adopt the techniques provided by society through practice and instruction, with other people guiding their activities. Several theoretical frameworks are presented, drawn from Biology, Ethology and Ecology. Our reflection about the problem is close to the theoretical perspective that emphasizes the sociocultural dimension of the context. In this sense, the social context is part of cognitive development. This approach to cognitive development emphasizes that cognition is an event or an activity integrating person and context.

Vygotsky concluded that social interaction with adults or more capable peers can enable children to solve new problems with assitance before they can solve them alone. Vygotsky called this difference between what a child can do alone and what she or he can be led to do with assistance the zone of proximal development. Hence the adult is very important in the learning process of the child. Several authors have attempted to show that adultchild interaction is a necessary precursor to strategic skill in young children. The research we have review allows us to conclude that the adult does not only improve children' learning in specific tasks but facilitates more complex processes in which the child learns to learn. These general abilities are difficult to describe because the authors refer to them by different terms and because different processes can have similar functions that help the child's learning in situations of problem-solving.

Language, in the Soviet framework, constitutes an essential element for explaining the influence of the adult in relation to the cognitive processes of the child. In the development language first comes pre-intellectual speech; and second by completely internal speech. Between them is private speech. There are many studies that have attempted to analyze the impact of private speech in the performance of a task, but the results are not always in agreement. Nonetheless, Wertsch (1979) argues that the social interaction can generate cognitive processes very similar to the processes that help private speech to emerge and enable learning in problem-solving.

The main question is to determine whether the relationship between child and adult can generate in the child cognitive or linguistic processes with similar functions to these of private speech. These functions in problem solving tasks are related to the control and planning of behavior and, 
also to the organization and the coding of the task. From this point of view we can argue that there are three kinds of research concerning the analysis of adult-child interaction.

1) The first tells us that interaction plays an important role in the transition from other-to self-regulation. Self-regulation refers to successful problem-solving behavior carried out independently. But in relation to the role of adults facilitating children's metacognitive processes, the results of research are not always in full agreement. Wertsch and Hickman (1987) analize some interactive processes whereby adults regulate young children's problem solving activities. They suggest that adult-child interaction improves children's performance because adults provide them with otherregulation which allows children's to become aware of the functional significance of the actions they executed. However Kontos (1983) considers that metacognitive processes can emerge from both adult assistance and solitary persistence at a problem solving task.

2) Other researchers analyze the role of the context in planning, mainly adult-child, and child-child interaction. Rogoff and collaborators (Rogoff, Gauvain and Gardner, 1987) suggest differences among children in planning. Older children plan in advance and apply planning strategies more flexibly to the circumstances of individual problems. They propose that more capable peers and adults may be influential in guiding the development of children's planning skills.

3) Finally, a third field of studies can be identified. For example, Ellis and Rogoff (1986) show how interaction with adults enable children to have the necessary information to solve tasks when, it is related to the structure of the problem. In this sense, adults enable the child to improve his/her coding and organization processes relevant for the task. 